\title{
3D Resistivity Imaging of Buildings and Foundations in Urban and Protected Areas
}

\author{
Fernando Almeida ${ }^{1}$, Manuel Matias ${ }^{1}$, Nuno Barraca ${ }^{1}$ and Rui Moura ${ }^{2}$ \\ 1. Department of Geosciences, University of Aveiro, Aveiro 3810-193, Portugal \\ 2. Department of Geosciences, Environment and Spatial Planning, University of Porto, Porto 4169-007, Portugal
}

\begin{abstract}
The use of Geophysics in Civil Engineering is an established practice. Herein, it will be exploited the use of 3D resistivity imaging to investigate foundations and features under buildings in urban areas where no direct invasive methods are allowed. High-resolution 3D resistivity requires the deployment of a dense grid of electrodes and, in urban areas, these methods face problems such as space limitation and availability owing to buildings, pavements, asphalts, obstacles and infrastructure. Thus, the use of conventional electrode arrays and grids may not be possible and alternatives must be sought. Herein a non-conventional array, pole-pole, is explored to characterize foundations and features under existing constructions in delicate and protected environments. A simple but effective data check is also proposed and allows to estimate the resistivity distribution in the area. Results prove the adequacy and versatility of the pole-pole array to carry out 3D resistivity surveys in urban areas. Data quality checks also proved effective and provided early qualitative images of the resistivity distribution in the areas to be used as an aid in later interpretation and data modelling stages.
\end{abstract}

Key words: Resistivity, 3D, pole-pole, urban, quality.

\section{Introduction}

The use of Geophysics in Civil Engineering is a well-established practice $[1,2]$. Recently there is an increasing interest in the restoration, renovation and rehabilitation of ancient buildings and monuments, often protected as local, national or world heritage. In these cases, building rehabilitation must conform to strict regulations aiming to preserve their features. This interest also extends to Modern and Contemporary buildings, landmarks of important architectural styles from those periods. Rehabilitation projects are an increasing activity in urban renewal and demand as much information as possible on the buildings about to be intervened. The most common way to obtain this information is through preliminary surveys using invasive/destructive methods. However, this approach is expensive, time-consuming and very likely to significantly damage or alter building structural or

Corresponding author: Manuel Matias, Ph.D., research field: applied geophysics. cultural/architectural features. Hence, there is a need for indirect, non-invasive, high resolution and fast technologies that can produce accurate images of the buildings and the information needed for a reasoned rehabilitation project.

The need to image the geological settings, building foundations, characteristics, pathologies and infrastructure location, among other targets, has led to the application of Geophysics to urban areas, the development of the so called Urban Geophysics [3, 4] and the use of high resolution resistivity imaging is common in these applications.

However, the application of resistivity imaging methods in urban areas can be difficult. Among other factors, limitations of urban resistivity geophysical surveys range from environmental noise, space availability, ground occupation, insulated ground conditions, since buildings, houses, pavements, asphalt or other types of obstacles can prevent the use of conventional linear electrode arrays or the use of a dense grid and parallel lines of electrodes. Field 
limitations can be even worse if the aim of the survey is to image the ground under existing constructions.

Nevertheless, resistivity imaging methods, in $2 \mathrm{D}$ or 3D mode, have been applied with success in the resolution of problems, such as, geotechnical engineering, detection of tunnels and fractures, investigating soils conditions under buildings and characterization of buildings and historical monuments [5].

Resistivity survey limitations are not restricted to the type of electrode array only, but also to the nature of the electrodes in use. Electrode arrays must be adapted to the available space and electrodes designed to operate in delicate areas where no physical or chemical damage can be inflicted to the surfaces and materials where they operate. This is particularly important when surveys are carried out in monuments or in protected buildings [6].

In the last decades, the development of automated and programmable equipment allowed the acquisition of large amounts of data and the design of field operations with non-conventional arrays. Thus, nowadays it is possible to operate with arrays of different geometries and choose the setups that are best suited to fulfil the aims of a particular survey $[7,8]$.

The need for higher resolution in resistivity response has led to the development of 2D and 3D techniques [9] and of non-conventional electrode arrays, such as the L, Corner arrays $[5,10,11]$ and the pole-dipole array [12]. In some cases, it is necessary to deploy the electrodes on the perimeter of the building or of the area of interest that is, wrapping the space with electrodes [13]. However, this particular set up limits the electrode arrangements used in the resistivity survey [8].

Urban surveys are usually carried out on surfaces where electrode contacts are often far from being optimal and data quality checks, based on the traditional principles of superposition and reciprocity [14] are difficult if not impossible to conduct because of the electrode arrays geometry.

Nowadays, checking on contact resistances is possible with modern field equipment but data quality checks still pose difficulties. Furthermore, in the case of high impedance electrical contacts it is often recorded a long term potential decay and, in these circumstances, the automatic interchange between current and potential electrodes may led to field measurements instability and errors that will interfere with modelling.

Although present computing and software facilities enable the acquisition of large amounts of data, the acquisition itself should not be too long in order to maintain suitable and constant electrode contacts throughout the survey and to keep operations within practical and economic standards.

One the other hand, it must not be forgotten that urban surveys and, in particular, surveys in buildings and monuments, should be done as quickly as possible to avoid disturbance to visitors and public in general.

Thus, there is a legitimate need to pursue a compromise between the number of measurements and the resolution of the survey. Back in the late 20th century Loke and Barker [15] proposed a data acquisition scheme, named "cross diagonal survey" based on the pole-pole array, to decrease the number of observations while maintaining an adequate resolution and quality of modelled data in resistivity imaging. The pole-pole array is a particular set up of electrodes from which any electrode configuration can be derived and, hence, it can be very flexible to adapt to the detection of shallow structures [16] and to urban and archaeological geophysics studies $[6,17]$.

However, some disadvantages have been referred about the use of the pole-pole array [18]. This array might run into practical problems in finding appropriate locations for B and N (Fig. 1), as these electrodes must be distant from the A, M electrode pair. Another problem is the possibility of recording telluric noise as the electrodes $\mathrm{M}$ and $\mathrm{N}$ are far apart.

Further comparison with more conventional arrays [18] indicates that the pole-pole array has poorer resolution, but deeper investigation depth and wider 


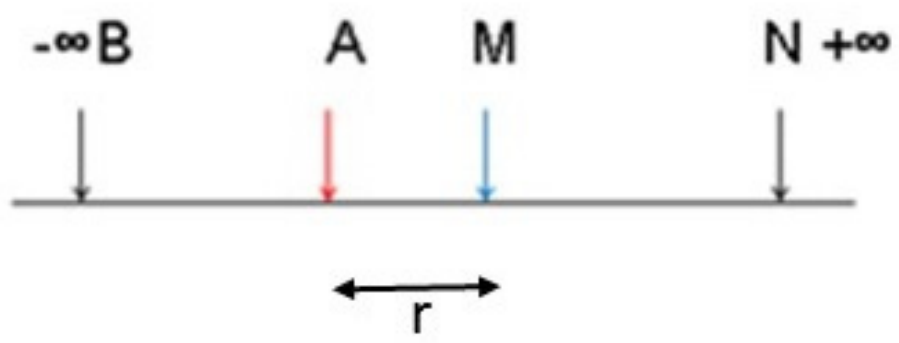

Fig. 1 The pole-pole array.

horizontal coverage.

It is the propose of this work to explore the application of the pole-pole array to small scale building foundation projects, in an urban environment, using different deployment array geometries.

It is also proposed to investigate a method capable of assuring field data quality and, thus, the overall quality of the data for later modelling and interpretation.

To accomplish these aims two case studies will be described. Resistivity data modelling, as any other geophysical data modelling, has a certain degree of ambiguity and uncertainty. To overcome this problem, modelling is often done using à priori information, when available.

The first study consists on the use of the pole-pole array around the perimeter of a contemporary building to investigate the ground beneath it. In this case, building foundations and infrastructure locations are known so this example is presented as proof of concept.

Since the suitability of the method was checked by the previous example, a second case study reports an investigation inside a monument where the electrode array is deployed around and inside the building to investigate foundations and the ground beneath it. This case refers to a World Heritage Monument and little direct or indirect information is available. Thus, resistivity modelling is supported by the experience obtained in the previous case and on the known engineering and architecture details of the monument. To further support resistivity modelling sensitivity analysis is also presented and discussed.
As modern resistivity field equipment allows the automated switching of current electrodes and potential electrodes a particular set up of the pole-pole array, the odd even array, was used. With this array, no current electrode is ever used as potential electrode and vice versa. Hence, any decay potentials, originated by high impedance electrical contacts, that might introduce noise in the data are simply avoided. On the other hand, this particular set up allows a faster coverage of the areas to investigate, diminishing the number of readings which, as mentioned before, is particularly important in public areas where operations must be carried out with minimum perturbation to the public and visitors. However, there is potential loss of resolution as the number of readings is diminished. Furthermore, the development of an innovative and simple application of electrical field potential theory is demonstrated to provide a tool to investigate the quality of large data sets for 2D/3D resistivity inversion.

This technique also allows estimating, for the first time, the resistivity at the current electrode positions and, thus, can provide an earlier tool for data interpretation, providing an early and initial model, before elaborate $3 \mathrm{D}$ inversion modelling is done, as demonstrated in the second case.

\section{3D Resistivity: The Pole-Pole Array}

The pole-pole array is a four electrode array where one current electrode, $\mathrm{B}$, and one potential electrode, $\mathrm{N}$ are placed far from the other two electrodes, that is, the current electrode $\mathrm{A}$ and the potential electrode $\mathrm{M}$ 
(Fig. 1.) Therefore, the array does not need to be either collinear or symmetric.

In practical terms, the ideal pole-pole array does not exist but a good operating approximation is possible if electrodes $\mathrm{B}$ and $\mathrm{N}$ are at distances larger than 20 times the largest separation between electrodes A and M. In these circumstances [18] the effects of the electrodes B and $\mathrm{N}$ in the measured values is less than $5 \%$, and thus a good approximation to the ideal pole-pole array is achieved.

The geographical location of the $\mathrm{B}$ and $\mathrm{N}$ electrodes is not important as long as distances are large. Nevertheless, the location of the electrodes must be chosen so that the physical conditions of the contacts are constant throughout the survey, that is, contact resistances must be stable during the period of operations.

If $r$ is the distance between the electrodes $\mathrm{A}$ and $\mathrm{M}$ (Fig. 1), the measured apparent resistivity, $\rho_{a}$, is calculated from:

$$
\rho_{a}=2 \pi r \frac{\Delta V}{I}
$$

where $\Delta V$ is the measured potential difference between electrodes $\mathrm{M}$ and $\mathrm{N}$ and $I$ is the electrical current intensity passed between electrodes A and B.

Several authors [18-21] have discussed the advantages and disadvantages of the pole-pole array, in particular, poor resolution at shallower depths, possibility of higher noise levels, as noise is proportional to $\mathrm{MN}$ distance, which is usually large in the case of the pole-pole array. However, the same authors also state that resistivity images are clearer, hence easier to interpret, than those provided by other arrays due to weak edge effects in pole-pole data.

Because of its geometry and of the superposition principle, any other array can be derived from the pole-pole array [21]. Therefore, pole-pole array data are easily compared with data from other arrays and can be used with any modelling software, provided the level of noise in the data is low.

If electrodes B and $\mathrm{N}$ are at the "infinite", that is, potential values at $\mathrm{M}$ do not depend on the influence of $\mathrm{B}$ and $\mathrm{N}$, measured values are plotted in accordance with Fig. 2 [21].

There are practical limitations to the use of the pole-pole array. To take advantage of the previous points and to minimize disadvantages, electrodes $\mathrm{B}$ and $\mathrm{N}$ must be at distances 20 times larger than AM, that is, distance $a$ cannot be more than a few metres to avoid practical problems in locating the electrodes $\mathrm{B}$ and $\mathrm{N}$. Thus, this array is particularly suited to small 2D/3D surveys using array spacings $r$ up to a dozen metres.

Nowadays 2D and 3D resistivity surveys are carried out using programmable resistivity meters, multicables and, therefore, the whole sequence of measurements is carried out automatically.

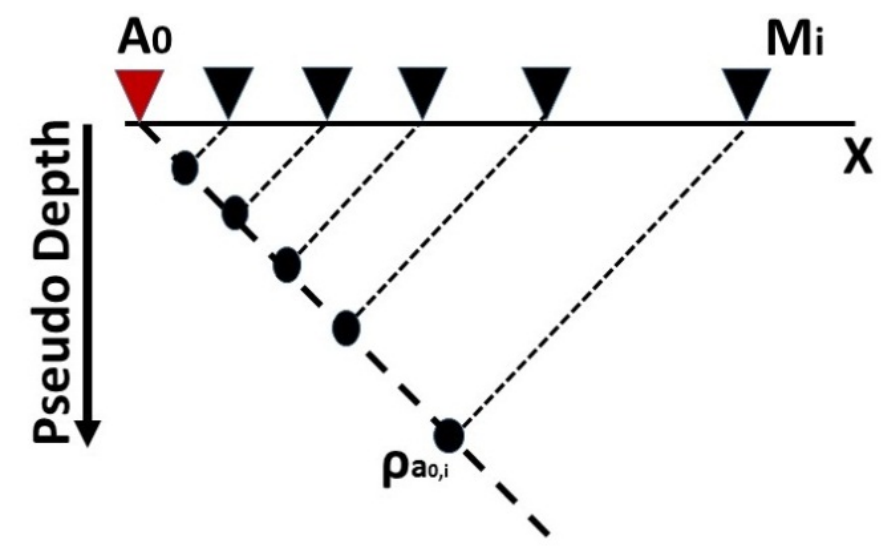

Fig. 2 Point of assignment of the resistivity values measured with the pole-pole array. 
During the measurements, as the electrodes are commuted, in one measurement, an electrode can act as a potential electrode and, soon afterwards, it can act as current electrode and vice versa. Therefore, the possibility of decaying potentials near the electrodes cannot be discarded during a whole sequence of readings and, if this occurs, extra and unwanted noise is added to the data. This is particularly important when working with high impedance electrical contacts as is often the case when using electrodes directly in contact with hard rock or highly resistive surfaces. This could be very important when using the pole-pole array as the noise increases with increasing MN distances.

To avoid such a problem, herein is proposed the use of the odd even pole-pole array $[6,17]$ to minimize the influence of decaying potentials that might exist and contaminate the signal during the commutation of the electrodes (Fig. 3).

In the odd even pole-pole array set up (Fig. 3), the odd numbers positions (red) are used as current electrodes points only, whilst the even numbers positions (blue) are used solely as potential electrode points. With this particular set up better quality data are obtained as eventual decaying potentials are not recorded since there are no electrodes that act as current electrodes and afterwards as potential ones and vice versa. For a setup of $2 n$ electrodes each current electrode provides $n$ readings and thus an overall of $n^{2}$ measurements are obtained for a complete survey.

\subsection{D Resistivity Data Processing}

To ensure data quality it is necessary to perform some field data checks. Traditionally superposition and tripotential theory [14] provides good checks on data quality. These checks are difficult, if not impossible to carry out, in the case of the pole-pole array because of its particular geometry.

Thus, a different and innovative approach is proposed to test field data quality obtained from the use of the pole-pole array. From electrical potential theory, the electric potential $V$ around a current point of intensity $I$ in a half space with resistivity $\rho$ decays with distance $r$, in accordance with the equation:

$$
V=\frac{\rho I}{2 \pi r}
$$

Therefore, the measured resistance $R(\mathrm{ohm})$ is given by

$$
R=\frac{V}{I}=\frac{\rho}{2 \pi} \times \frac{1}{r}
$$

That is, if the measured resistance, $R$, is plotted against the inverse distance between the current electrode and the potential electrode, $1 / r$, the relation is described by a straight line with an angular coefficient $\rho /(2 \pi)$, where $\rho$ is the resistivity of the ground under investigation. In a homogeneous isotropic medium $\rho$ is constant. However, as heterogeneities are expected this value can vary with electrode location.

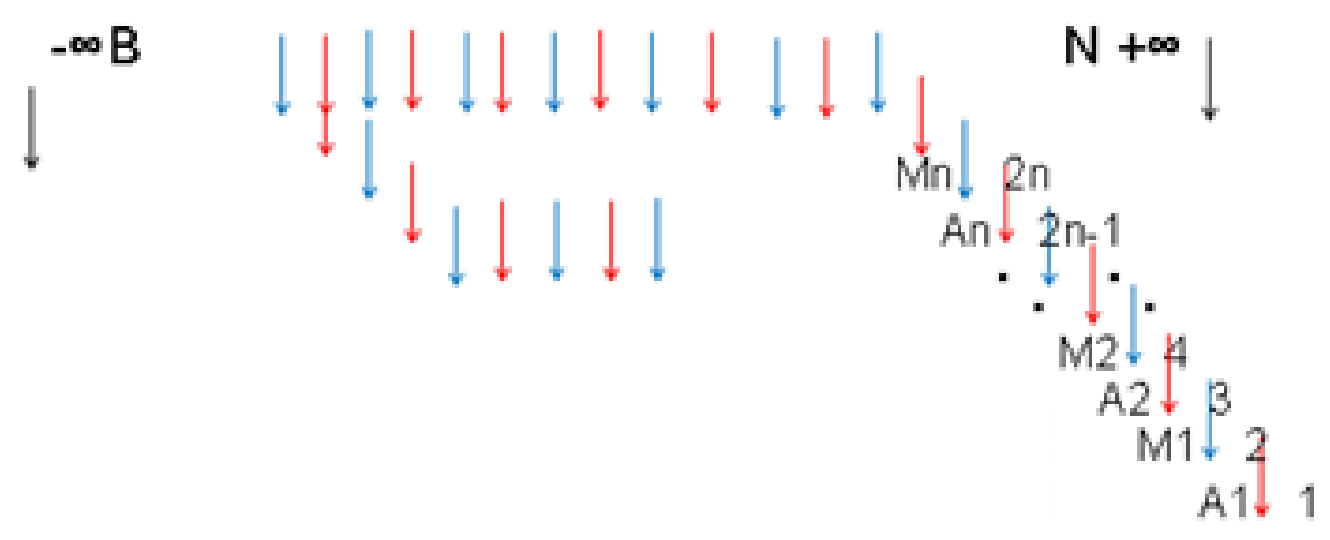

Fig. 3 The odd-even pole-pole array (red arrows/odd-current electrodes; blue arrows/even-potential electrodes). 
As pole-pole array measures the potential difference between electrodes A (current electrode) and $M$ (potential electrode) (Fig. 1), it is possible to construct a graph showing the relationship between $R$ (electrical resistance) and $1 / r$ Eq. (3), where $r$ is the distance between electrodes A and M (Fig. 1).

Furthermore, if data are collected in a 3D manner it is also possible to construct potential decaying surfaces from the field data, as the positions of electrodes A and $\mathrm{M}$ are distributed over the whole area to investigate.

Therefore, for each current electrode position, A, it is possible to construct a graph depicting the relation between the measured resistance, $R$, and the inverse distance, $1 / r$, between A and each position for the potential electrode $\mathrm{M}$.

This relation must be described by Eq. (3) and large deviations, if any, are considered as measurement errors, arising from bad contacts, electrodes displacement, among others factors. Therefore, once field data are plotted a robust linear correlation [22] is carried out between the measured resistance, $R$, Eq. (3), and the inverse distance, $1 / r$, for each current and potential electrode pair, left of Fig. 4 . Then points that do not fulfil the decaying function are removed or, alternatively, an acceptance quality threshold can be established. The threshold level, a percentage of the estimated resistance values, is defined by the operator and can be adjusted. As an example, on the left of Fig. 4, the green dots are good quality measurements but the blue cross represents an erroneous reading. The computed regression line between resistances and inverse distance between the current and potential electrodes also allows an estimate of the resistivity at the current point. In fact, as mentioned before, from Eq. (3) the angular coefficient of the straight line gives the value of the resistivity divided by $2 \pi$. Thus, it is possible to compute the resistivity value at the current point.

On the right of Fig. 4, the decay function Eq. (3) is shown for a space around current electrode A. In this figure, higher values (red/brown) are depicted around the position of the current electrode A, whilst low values (dark blue) are shown away from the electrode. The gradation of colours shows the decay effect of the resistances, as expected from Eq. (3).

Once the resistivity values at the current points are
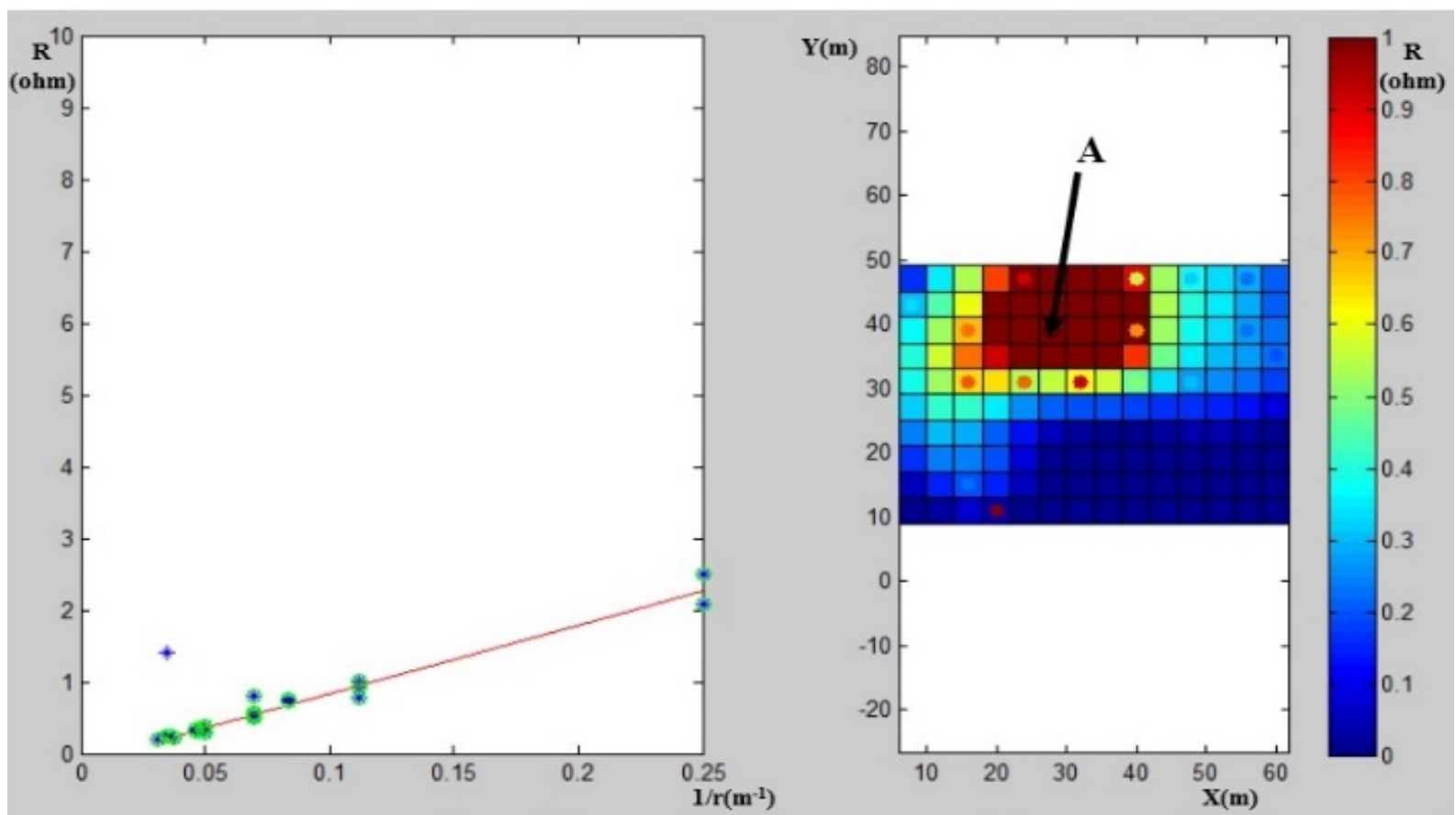

Fig. 4 Regression between the measured earth resistance and the inverse distance for each current, potential electrode pair (left); decay surface for a 3D survey around electrode A (right). 
estimated it is possible to construct a map depicting the resistivity at all the current points in the investigated area, as it will be shown in the case studies. Such a map can provide an early and initial resistivity model for future 3D resistivity inversion and modelling.

\section{Case Studies}

The use of the pole-pole array in building and foundation investigations will be addressed in two different field situations that correspond to different electrode geometries of the pole-pole array. In fact, this array has the versatility to be used with different geometries in accordance with space availability, as well as, the survey objectives. Resistivity data will be modelled using the widely used and successfully tested and widely used software Res3DInv [18].

In fact, there are countless examples in the literature of application of this software. Nevertheless, it is known that resistivity data modelling, as any other geophysical data, is ambiguous and proposed solutions are not unique. Therefore, models are presented together with "a priori" information about local ground conditions and known construction characteristics.

Thus, ambiguity in the final interpretation is largely reduced and confidence in the proposed models is boosted.

In the first case, the pole-pole array was deployed around a contemporary building showing differential settlement, as it was not possible to place any electrodes inside the building due to the pavement and the building use (Fig. 5). As the locations of building foundations and other infrastructure are known, this case will be used as a test and proof of concept example. The second case consists on the study of the ground beneath the main Nave (Church) of the protected monument. In this case, pole-pole array electrodes were deployed both around and inside the building proving the flexibility and applicability of the array.

\subsection{The Geosciences Department, University of Aveiro}

The Department of Geosciences building (Fig. 5), was constructed in the end of the 20th century, over recent sedimentary formations, that is, Quaternary sands, about 5 metres thick that lay over clayey marls.

The building shows some signs of structural settlement as well as high degree of humidity in the Northern corner.

Building foundations consist on circular concrete shafts linked by a structure of lintels that penetrate at least $0.50 \mathrm{~m}$ into the ground (Fig. 6).

Since the Geosciences building constructing characteristics were known beforehand this field case intends to explore the use of the pole-pole array deployed around the area and to test 3D inversion modelling in the investigation of the construction impact, excavation, foundations, infrastructures and seepage beneath the building.

Field data were processed and quality checked in accordance with the procedure described previously. Once electrode resistivities were estimated, they were assigned to the corresponding electrode positions and are shown in Fig. 7. In this figure, the orange/red dots correspond to high resistivities whilst, blue dots correspond to low resistivities. This figure provides an
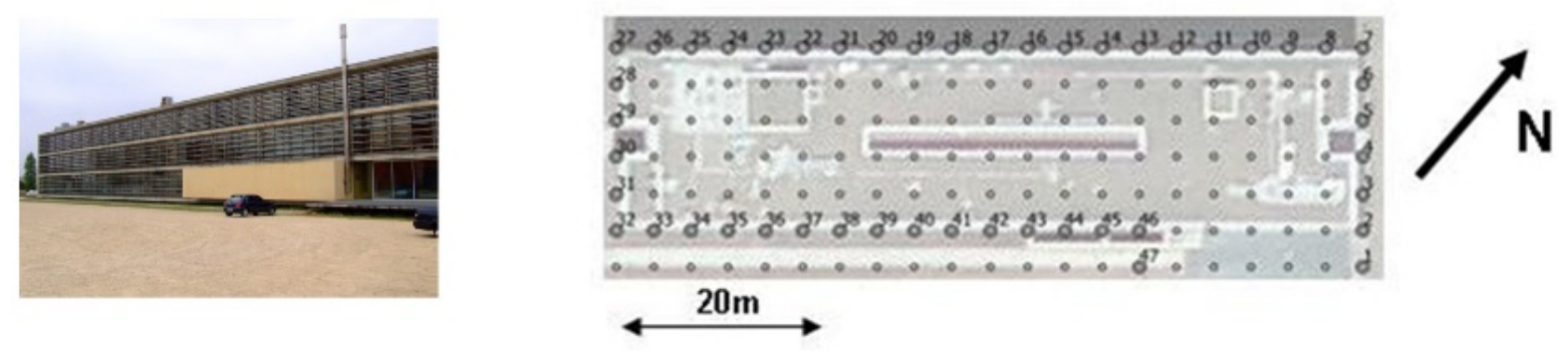

Fig. 5 The Department of Geosciences, University of Aveiro (left). Location of the pole-pole electrodes (right). 


\section{LAYER 1 DEPTH 0.00-0.50m}

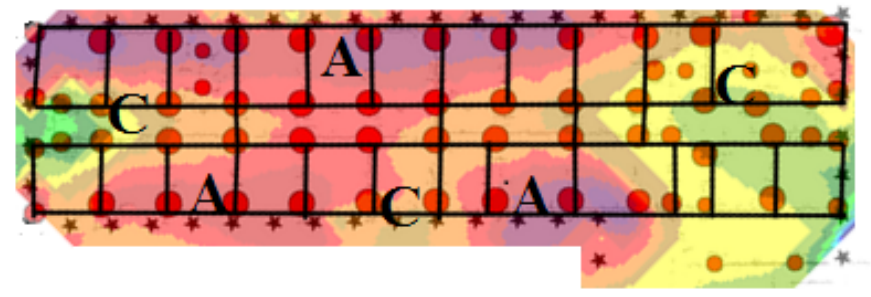

Fig. 6 Geosciences building foundations: lines-lintels; red circles—concrete shafts. Background resistivity model (depth 0-0.5 m); red—resistive, green/yellow—conductive).

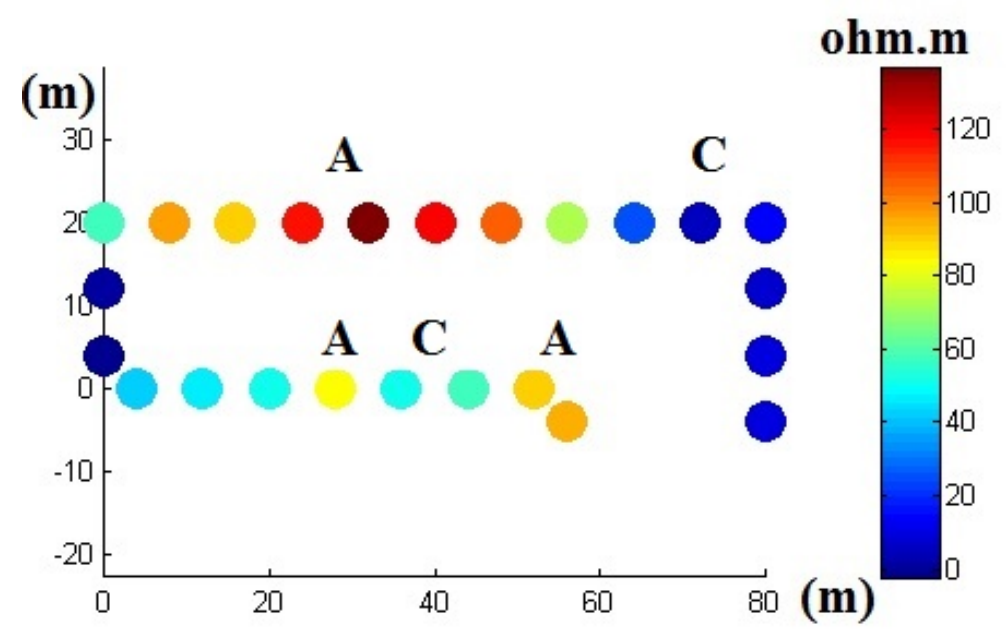

Fig. 7 Estimated resistivity at the current electrodes around the Department of Geosciences (Uni. Aveiro).

overall spatial view of the resistivity distribution in the area. As depicted in Fig. 6, in broad terms the geometry of the resistivity contour lines matches the geometry of the foundations. There are regions of higher resistivity- $\mathrm{A}$ - that correspond to the expected position of the building foundations.

On the other hand, the blue dots correspond to lower resistivity areas, $\mathrm{C}$, that coincide with the accumulation of moisture and water in the building.

The 3D inversion of the resistivity data was performed using Res3dInv [18] and the models for different depths are depicted in Fig. 8. The largest rms error is $21.5 \%$ after nine iterations.

At the shallower depths, less than $4.00 \mathrm{~m}$ (two top models and second row left) the impact of the building, and its foundations, is sharply depicted by the higher resistivity regions, A. These regions correspond to the foundations along the longer walls and lintels. Further fainter resistive anomalies, A1, correspond to foundations oriented at $90^{\circ}$ to the previous foundations. It must be pointed out that the resistive anomaly A, positioned in the top right of the shallower map, corresponds to the location of an amphitheater built at a lower level. However, the two top models also show conductive regions, $\mathrm{C}$, which seem to interrupt the expected foundations location. These can be interpreted as defects or severe damage on building foundations where groundwater can flow easier.

At larger depths, two lower models, a highly conductive area $\mathrm{C}$, should correspond to Plio-Pleistocene channels that are known to exist under the building. Such features must accumulate a large quantity of water, thus damaging the foundations and contributing to the accumulation of moisture and water 

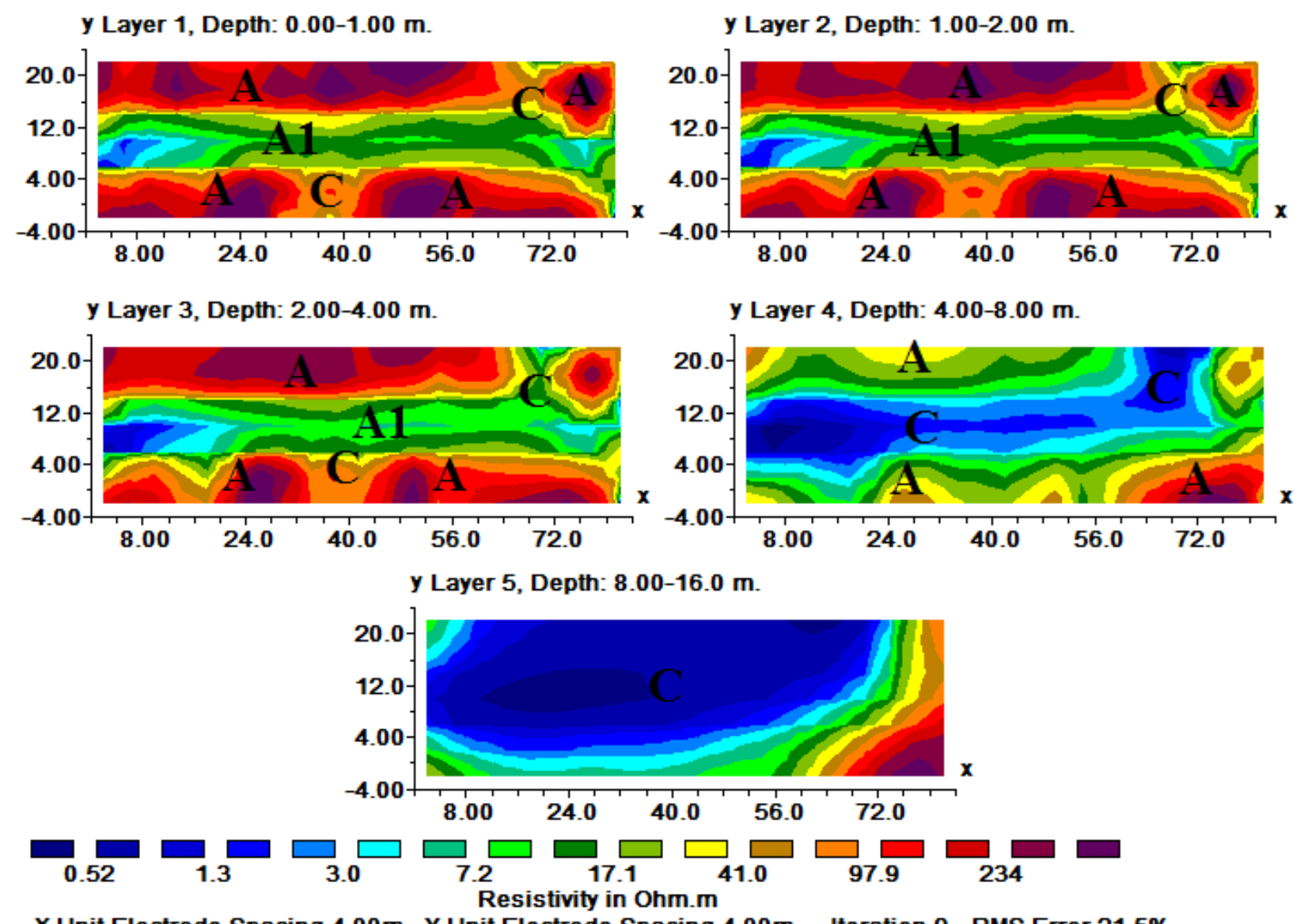

X Unit Electrode Spacing 4.00m. Y Unit Electrode Spacing 4.00m. Iteration 9 - RMS Error 21.5\%

Fig. 8 3D resistivity models (Dep. Geosciences).

in the basement of the building.

Sensitivity analysis of the models from Fig. 8 is shown in Fig. 9. This figure reveals higher sensitivity near the electrode locations as it should be expected because of the geometry of the array [18]. The highest sensitivity values calculated of around 6 , which is much lower than those indicated [19].

So, in this case resistivity models obtained from the use of the pole-pole array are clearly dependant of the location and design of the foundations of the building. Thus they provide a clear picture of their position as depicted by the resistive areas in Fig. 8. Conductive areas are consistent with areas of groundwater flow where no buried obstacles are found, that is between the longitudinal lintels, as it can be seen in Fig. 6.
3.2 3D Resistivity Survey in Batalha Monastery: The Nave

The Nave of Batalha Monastery, left of Fig. 10, is a 14th century UNESCO monument, located in central Portugal, lat $39^{\circ} 39^{\prime} 39^{\prime \prime} \mathrm{N}$, long $8^{\circ} 49^{\prime} 33^{\prime \prime} \mathrm{W}$, near the town of Leiria.

The Monastery was built on a valley where drift, few metres thick, covers a Jurassic limestone bedrock.

The odd even pole-pole array was used to investigate the ground below the monument and measurements were carried out with three sets of 47 electrodes. Electrodes where located both around the Nave, $4 \mathrm{~m}$ apart, as well as inside the Nave, with a grid of electrodes also $4 \mathrm{~m}$ apart, right of Fig. 10, using three sets of 47 electrodes. Thus one set was used in the central 
y Layer 1, Depth: 0.00-1.00 m.

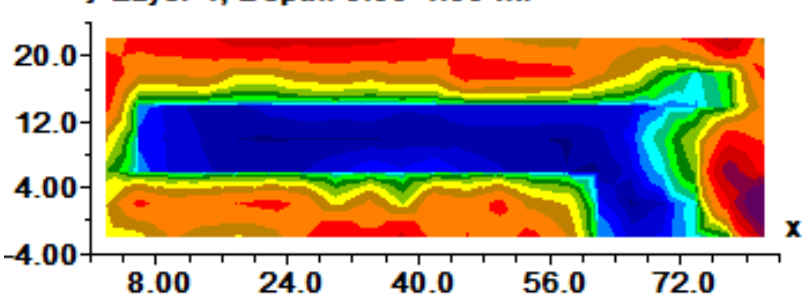

y Layer 3, Depth: $2.00-4.00 \mathrm{~m}$.

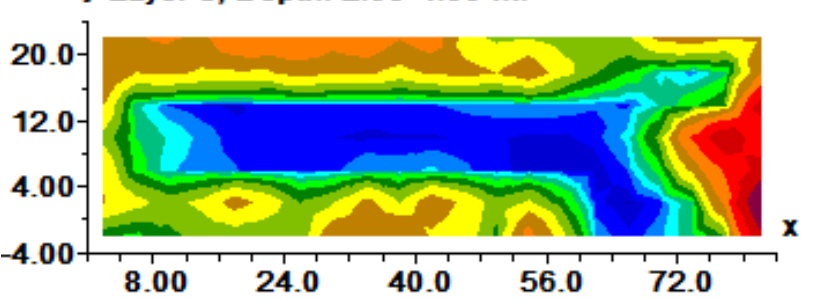

y Layer 2, Depth: 1.00-2.00 m.

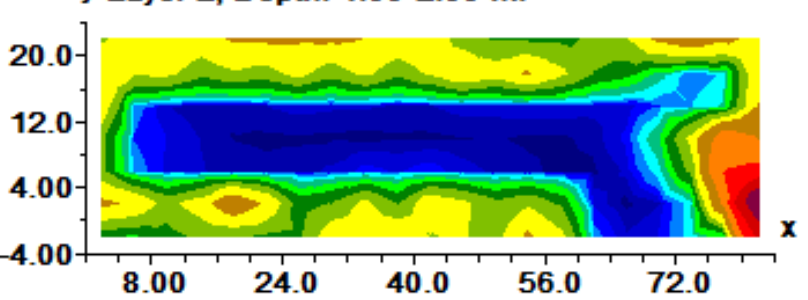

y Layer 4, Depth: 4.00-8.00 m.

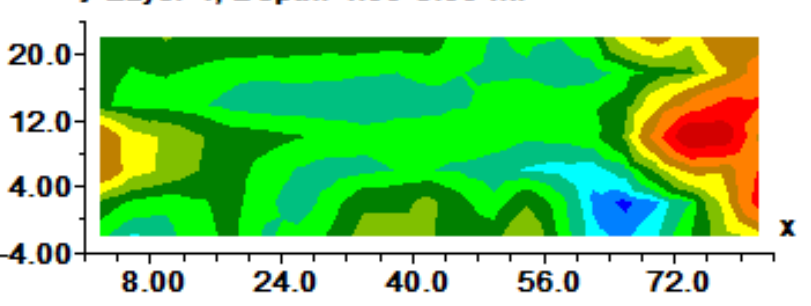

y Layer 5, Depth: 8.00-16.0 m.

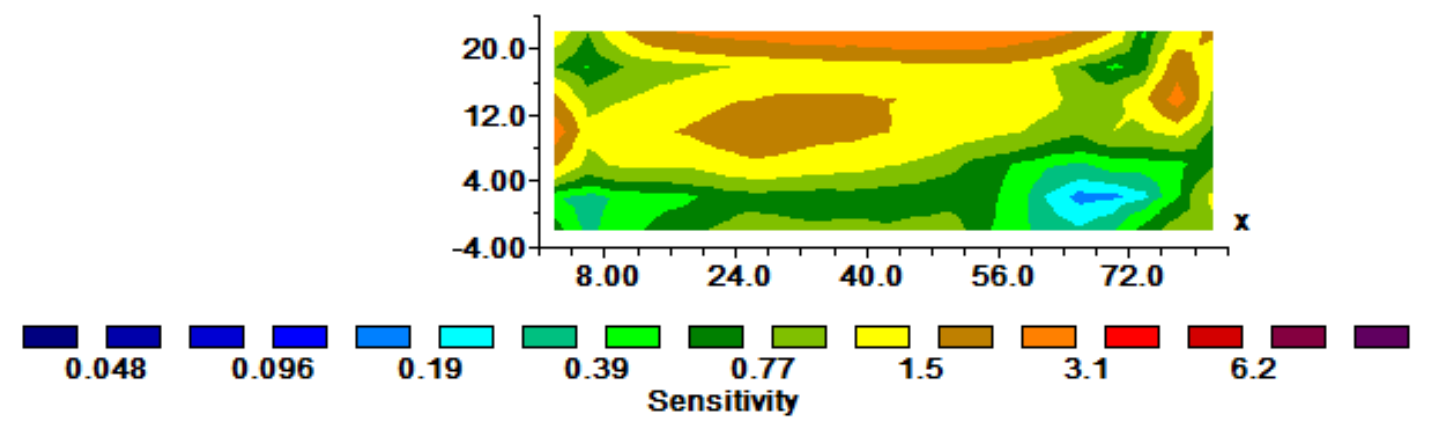

X Unit Electrode Spacing 4.00m. Y Unit Electrode Spacing 4.00m. Iteration 9 - RMS Error $0.00 \%$

Fig. 9 Sensitivity analysis for resistivity models in Fig. 8-Department of Geosciences.
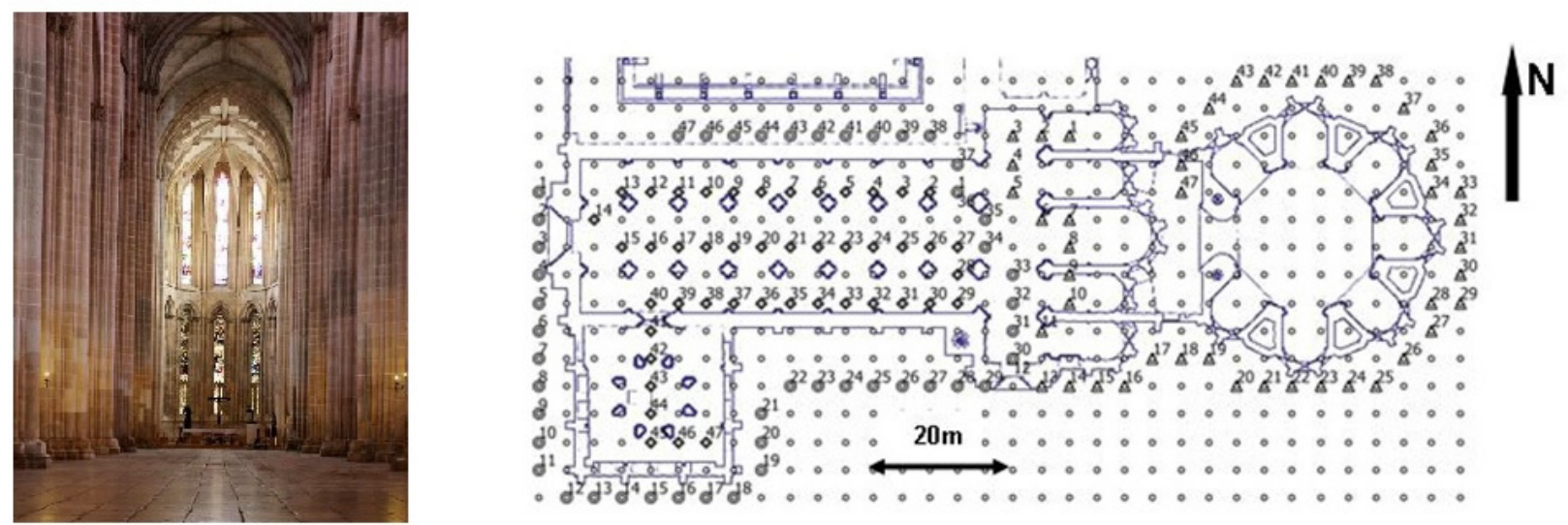

Fig. 10 The Nave, left, pole-pole measuring points in and around the Nave, right.

and left part of the monastery, another set on the right side and a last one inside the Nave itself.

Once the readings were done and data quality checks performed the estimated resistivity values at the electrode positions are shown in Fig. 11. In this figure, a more conductive zone (dark blue dots) towards the 
left is clearly separated from a more resistive area (brown, orange, green dots) by the black line in Fig. 11. The position of this black line coincides with the location of a visible fracture on the walls and ceiling of the Nave. The more resistive values are shown near the Joao I (King John I) cloister and in the location of the Nave transept-Location A. Zone B corresponds to a chapel added later to the Nave and built on an embankment.

The 3D modelled resistivity data are shown in Fig. 12. This figure shows five model depth slices obtained with Res3DInv. Therefore, Fig. 12 depicts, from top to bottom, models for depths from 0 to $16 \mathrm{~m}$. A maximum rms error of $20.3 \%$ was recorded after seven iterations.

In the upper sections, there is a contrast marked by the black line that crosses the models diagonally. This line coincides with the black line in Fig. 11. Direct comparison between Figs. 11 and 12 provides a very close qualitative interpretation of the data in the investigated area.

At the shallowest depths, the two top sections with depths varying from $0-1 \mathrm{~m}$ (top) and 1-2 $\mathrm{m}$ (second from top) show significant variations of modelled resistivities. Area A corresponds to resistive regions that occur near the transept of the Nave. In this region, the pressure of the structure over the columns is larger.

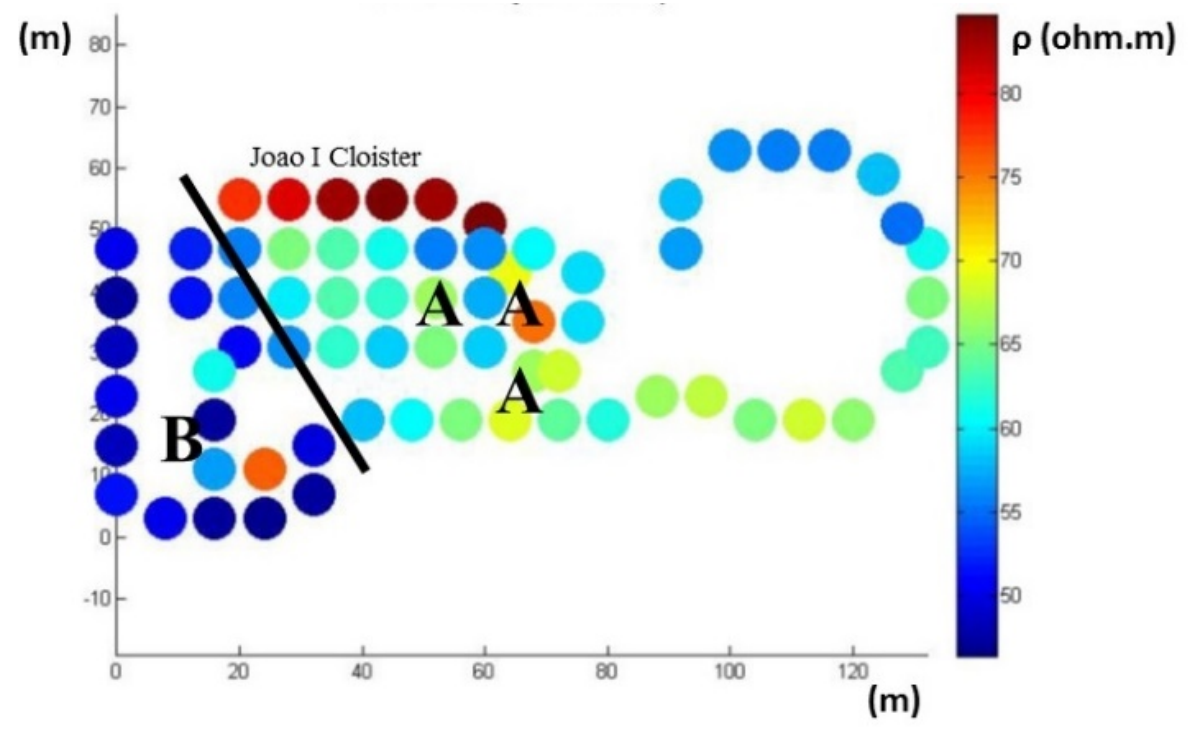

Fig. 11 Estimated resistivity at the electrodes in the Nave.
Hence, it is expected that the pressure over the ground is also larger and soil compaction must increase, diminishing porosity. On the other hand, it is also expected that foundations are stronger in this region.

Zone $\mathrm{B}$ is a more conductive area and corresponds to the Founders Chapel location. This building, later added to the Monastery, is a lighter structure and, according to history, was built over an embankment. In these conditions, it is expected that resistivity model values are lower than the previous ones.

Sensitivity data for the models in Fig. 12 are depicted in Fig. 13. As shown, sensitivity is higher when ground coverage is larger as it is the case of the Nave. To the right, where there are few electrodes sensitivity is shown to decrease, right part of Fig. 13. However, calculated sensitivity values are lower than 3 , which is much lower than those indicated in theoretical works [19].

Therefore, 3D resistivity mapping in the Nave revealed that the most resistive areas correspond to the regions nearer to the transept where pressure on the ground must be higher and foundations stronger. The Founder Chapter location corresponds to a lower resistive region confirming its lighter structure and that it was built over less compact soil. 
y Layer 1, Depth: 0.00-1.00 m.

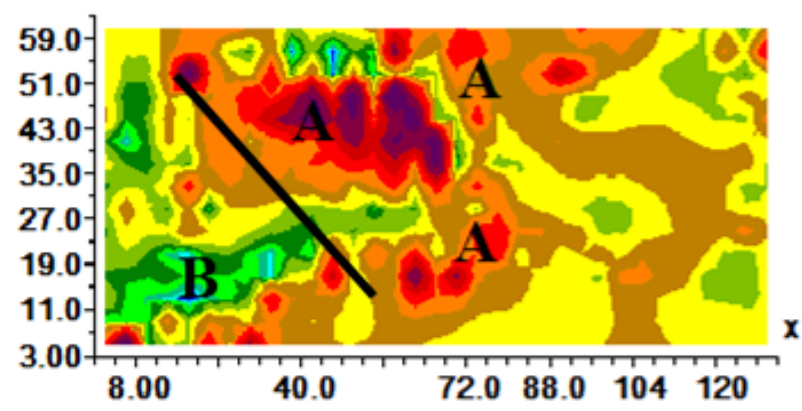

y Layer 3, Depth: $2.00-4.00 \mathrm{~m}$.

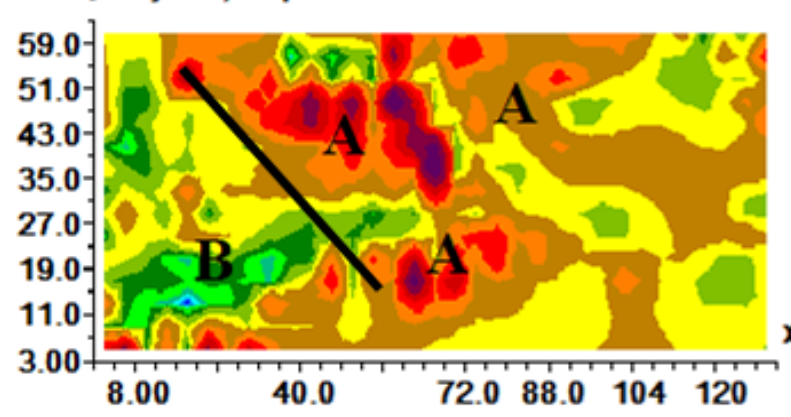

y Layer 2, Depth: 1.00-2.00 m.

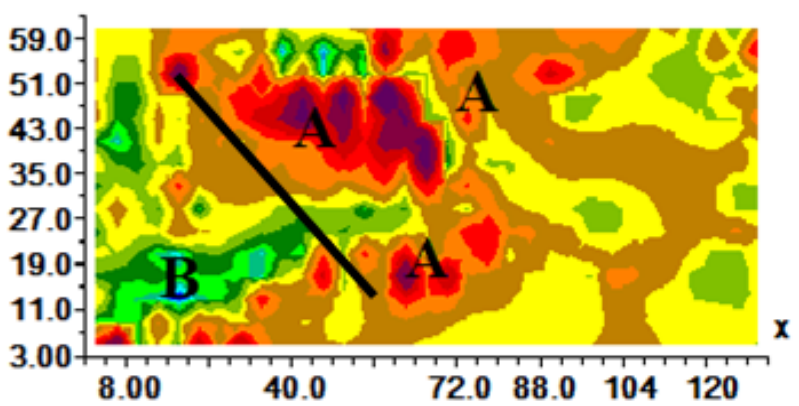

y Layer 4, Depth: $4.00-8.00 \mathrm{~m}$.

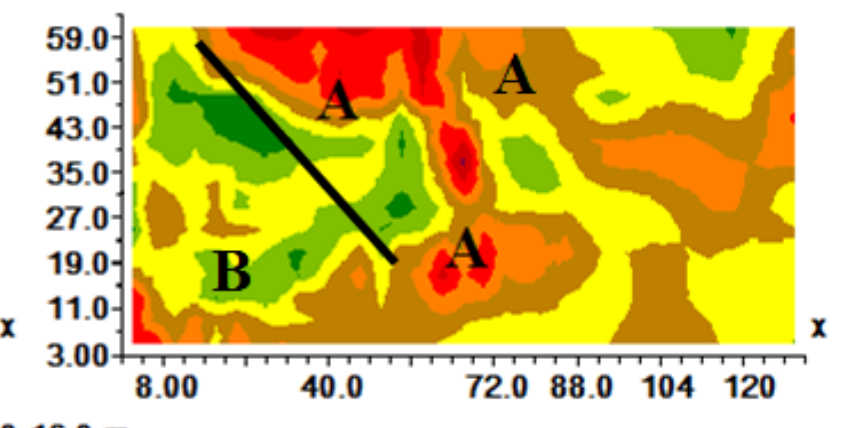

y Layer 5, Depth: 8.00-16.0 m.

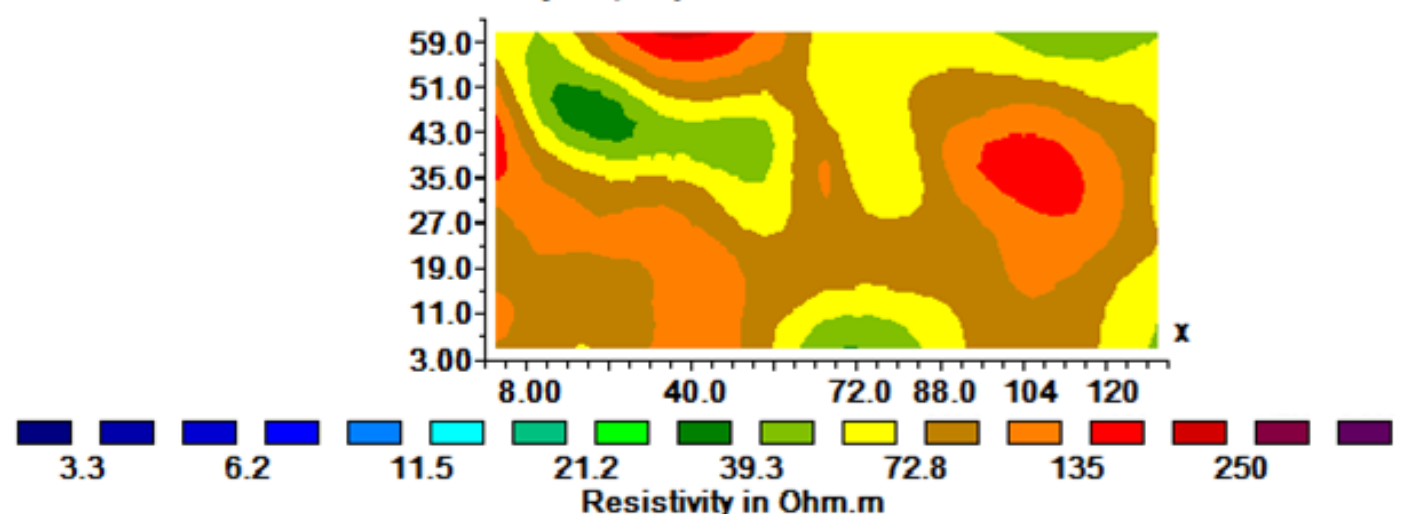

X Unit Electrode Spacing 4.00m. Y Unit Electrode Spacing 4.00m. Iteration 7 - RMS Error 20.3\%

Fig. 123 D resistivity model in the Nave. 
y Layer 1, Depth: 0.00-1.00 m.

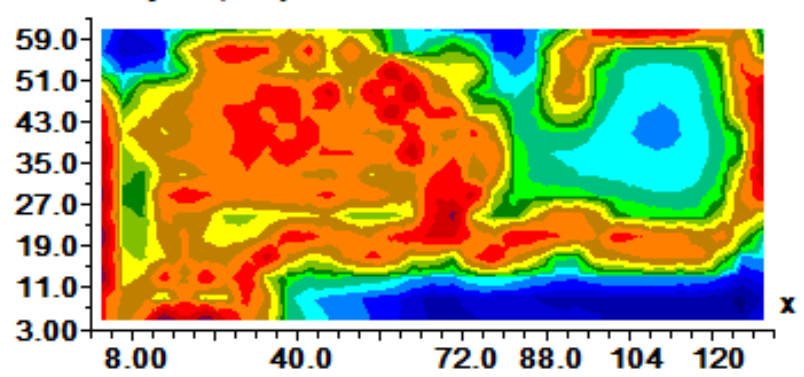

y Layer 3, Depth: $2.00-4.00 \mathrm{~m}$.

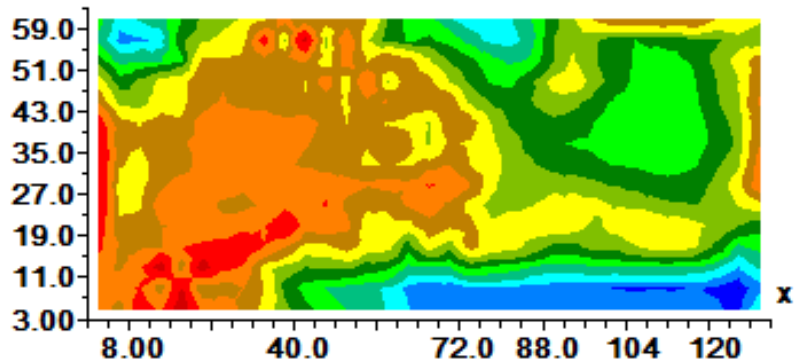

y Layer 2, Depth: 1.00-2.00 m.



y Layer 4, Depth: 4.00-8.00 m.

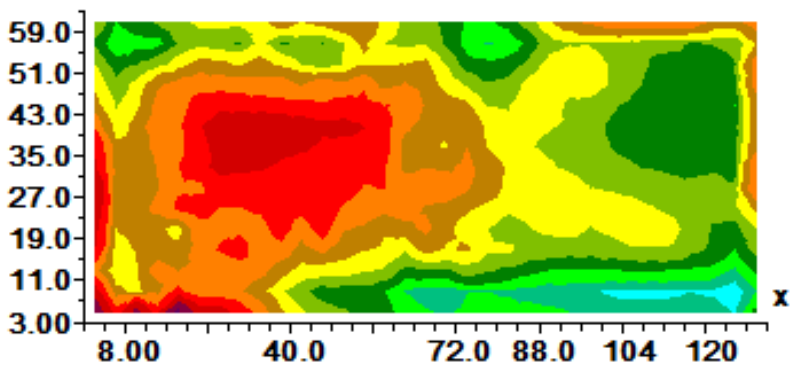

y Layer 5, Depth: 8.00-16.0 m.

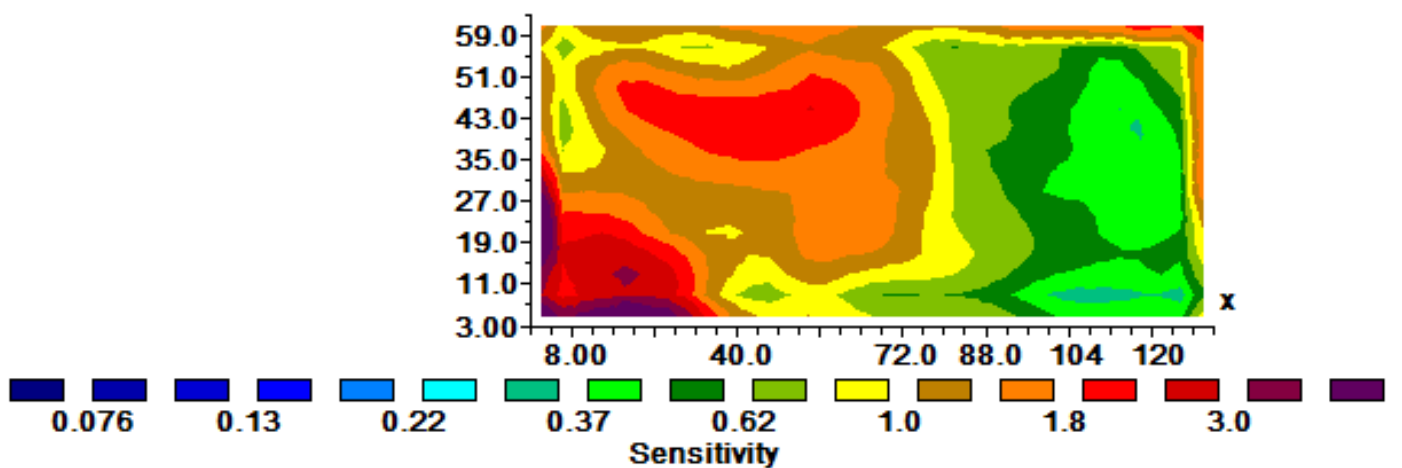

X Unit Electrode Spacing 4.00m. Y Unit Electrode Spacing 4.00m. Iteration 7 - RMS Error $0.00 \%$

Fig. 13 Sensitivity values for resistivity models in Fig. 18 - the Nave.

\section{Conclusions}

The pole-pole array is a non-conventional array with known advantages and disadvantages. The objectives of this study were to investigate its use in small scale, urban surveys, detailing its virtues and developing data processing strategies capable of checking data quality and, simultaneously, enhancing the information provided by its use.

It is clear that the pole-pole array can be used in urban areas with success and advantage. Owing to its versatility, it is possible to deploy the electrodes both around and inside the areas to investigate, where it is necessary or possible, which is a clear plus over linear, $\mathrm{L}$ and dipole-dipole arrays. Furthermore, as any other array can be derived from the pole-pole array, electrodes can be deployed using the most convenient geometry and the most suitable spacing $r$.

Thus, when compared with other more conventional arrays, the pole-pole array favours the optimization of the obtained information with space availability and the aims of a particular survey. However, in practice, it must be considered that distance MN (Fig. 1) must be small in order to obtain a good approximation to the theoretical pole-pole array. This restricts the dimensions of the areas to investigate but, in most 
cases, they are within the objectives of urban surveys.

As demonstrated, pole-pole array data can be easily checked for quality and the developed procedure allows the estimation of resistivity at the current points. Furthermore, it was proved that estimated resistivity maps can be used as an earlier source for qualitative interpretation.

Finally, as the pole-pole array is the basis of any electrode array, thus, array geometry and field measuring sequences can be easily introduced in modern programmable resistivity meters, and data can be readily imported to existent software for resistivity model inversion.

Future work should include modelling using bodies of known geometry to provide further analysis, interpretation and comparison with other arrays. Furthermore, comparison between pole-pole data and models with other high-resolution geophysical methods, such as Ground Probing Radar, could also be important to validate and compare field solutions and modelling.

\section{Acknowledgments}

The authors wish to acknowledge the contribution of the Batalha Council, the workers of the Monastery of Batalha, Morph Lda. (Coimbra) for lending some field equipment and the Portuguese Science and Technology Foundation, project UID/GEO/04035/2013, for the financial support.

\section{References}

[1] Nazarian, S., Xiong, Y., and Rosenblad, B. 2007. "Innovative Applications of Geophysics in Civil Engineering." American Society of Civil Engineers (ISBN (978-0-7844-0908-4).

[2] Coutinho, R., and Mayne, P. 2012. Geotechnical and Geophysical Site Characterization. Leiden: CRC Press.

[3] Miller, R. 2013. "Introduction to This Special Section: Urban Geophysics.” Leading Edge 32 (3): 248-49.

[4] Benjumea, B., Merino, J., and Dalhin, T., ed., 2018. "NSG Special Issue on Urban Geophysics: New Developments and Research Trends." Near Surface Geophysics 16.

[5] Tejero-Andrade, A., Cifuentes, G., Chavez, R. E., Lopez Gonzales, A., and Delgado-Solorzano, C. 2015. "L Array and Corner Arrays in 3D Electrical Resistivity
Tomography: An Alternative for Geophysical Surveys in Urban Zones." Near Surface Geophysics 13: 1-13.

[6] Almeida, F., Carmine, P., Gonçalves, L., and Daniel, L. 2001. "Odd Even Pole-Pole Spread electrode Commutation in Resistivity 2D Cross Borehole and 3D Surface Imaging Porto/Portugal Underground Tunnelling Case." In Proceedings of the 7th EEGs meeting EGS101.

[7] Loke, M. H., Wilkinson, P. B., Uhlemann, S. S., Chambers, L. S., and Oxby, J. E. 2014. "Computation of Optimized Arrays for 3-D Electrical Imaging Surveys." Geophysical Journal International 199: 1751-64.

[8] Loke, M. H., Wilkinson, P. B., Chambers, J. E., Uhlemann, S. S., and Sorensen, J. P. R. 2015. "Optimized Arrays for 2-D Resistivity Survey Lines with a Large Number of Electrodes." Journal of Applied Geophysics 112: 136-46.

[9] Dahlin, T., and Bernstone, C. 1997 "A Roll-along Technique for 3D Resistivity Data Acquisition with Multi-electrode Arrays." In Proceedings of the Symposium on the Application of Geophysics to Engineering and Environmental Problems, 927-35.

[10] Chavez, G., Tejero, A., Alcantara, M. A., and Chavez, R. E. 2011. "The L array, a Tool to Characterize a Fracture Pattern in An Urban Zone.” Paper presented on Extended Abstracts of NSG 2011.

[11] Chavez, R.E., Cifuentes,G., Argote, D. L., and Hernandez, J. E. 2015. "A Special ERT-3D Array Carried out to Investigate the Subsoil of the Pyramid El Castrillo, Chichen Itza, Mexico." Paper presented on Extended Abstracts of We21B17, NSG 2015.

[12] Denchik, N., Marescot, L., and Chapellier, D. 2006. “3D Resistivity Monitoring-An Effective Electrode Array to Follow Preferential Flow during Rainfalls." In Near Surface-12th European Meeting of Environmental and Engineering Geophysics. DOI: 10.3997/2214-4609.201402685.

[13] Argote-Espino, D., Tejero-Andrade, A., Cifuentes-Nava, G., Iriarte, L., Farías, S., Chávez, R. E., and López, F. 2013. "3D Electrical Prospection in the Archaeological Site El Pahñu, Hidalgo State, Central Mexico.” Journal of Archaeological Science 40: 1213-23.

[14] Habberjam, G. 1979. "Apparent Resistivity Observations and the Use of Square Array Techniques." Geopublication Associates, Geoexploration Monographs 1 (9).

[15] Loke, M. H. and Barker, R. D. 1996. "Practical Techniques for 3D Resistivity Arrays and Data Inversion." Geophysical Prospecting 44: 499-523.

[16] Samadi, H., and Samadi, M. 2013. "The Application of Pole-Pole Array for the Detection of Shallow Structures Case of Studies South West Tehran Iran." World Applied Sciences Journal 27: 684-7.

[17] Almeida, F., Barraca, N., Moura, R., and Matias, M. 2016. "Odd even Pole-pole Array and 3D Resistivity Surveys in 
Urban and Historical Areas." In 22nd European Meeting of Environmental and Engineering Geophysics. DOI: 10.3997/2214-4609.201601965.

[18] Loke, M.-H. 1999. "Electrical Imaging Surveys for Environmental and Engineering Studies-A Practical Guide to 2D and 3D Surveys." Accessed February 20, 2018. www.heritagegeophysics.com.

[19] Roy, A., and Apparao, A. 1971. "Depth of Investigation in Direct Current Methods." Geophysics 36: 943-59.

[20] Brunel, P. 1994. "Dispositif Multiélectrode en courant continu. Etude et application i des structures bidimensionnelles." Ph.D. thesis, the University of Paris 6.

[21] Robain, H., Albouy, Y., Dabas, M., Descloitres, M., Camerlyinck, C., Mechler, P., and Tabbagh, A. 1999. "The Location of Infinite Electrodes in Pole-Pole Electrical Surveys: Consequences for 2D Imaging." Journal of Applied Geophysics 41: 313-33.

[22] MathWorks 2014. "Statistics Toolbox: User's Guide (R2014a)." Accessed January 29, 2018. www.mathworks.com/help/stats/robustfit.html. 